\title{
Chicken Production Systems and Market Oriented in Post-Conflict in DRC
}

\author{
Musale Mukuki Dieudonné Katunga1, Katunga Fidel Balemirwe ${ }^{2}$, Bahige Masheka ${ }^{1}$ \\ ${ }^{1}$ INERA Mulungu, Bukavu, DRC \\ ${ }^{2}$ Faculté de médecine vétérinaire, Université du cinquantenaire Lwiro, East of DRC, DRC \\ Email: stylonya@gmail.com, katungamusale@yahoo.fr, katungamusale@inera-rdc.org, fidelkatunga@yahoo.fr, \\ b.masheka@yahoo.fr
}

How to cite this paper: Katunga, M.M.D., Balemirwe, K.F. and Masheka, B. (2020) Chicken Production Systems and Market Oriented in Post-Conflict in DRC. Open Access Library Journal, 7: e6172.

https://doi.org/10.4236/oalib.1106172

Received: February 17, 2020

Accepted: April 21, 2020

Published: April 24, 2020

Copyright $\odot 2020$ by author(s) and Open Access Library Inc.

This work is licensed under the Creative Commons Attribution International License (CC BY 4.0).

http://creativecommons.org/licenses/by/4.0/

(c) (i) Open Access

\begin{abstract}
Livestock production in DRC is very low and cannot provide sufficient animal proteins and contribute efficiently to poverty alleviation. In this way, a survey was carried out on chicken's production in 7 sites in the country: Bukavu and his hinterland, Minova, Bweremana-Sake, Goma, Kinshasa, Lubumbashi and in Kongo central (Mvuazi-Kolo). Survey was conducted on 8th to 23rd March 2015 at the household and chickens company level using standard methods of interviews and structured questionnaires on chicken's production systems and commercialization. Results confirm that local chickens are yet commonly rearing in the country. They were eating by scavenging, rate of mortality was high, chicken accommodation was not yet well performed and the most disease was New Castle. Extension services had low involvement in the chicken's production. Prices were high in all the sites. This should be an opportunity for the businessmen to boost the chicken industry production in the country. This study aims to survey current chicken industry and the status of technology based on the Korea-Africa Food Agriculture Cooperation Agency (KAFACI) project on the promotion of good management for increased productivity of market oriented small-scale chicken producers in DRC.
\end{abstract}

\section{Subject Areas}

Agricultural Science, Food Science \& Technology

\section{Keywords}

RDC, Survey, Chickens, Production Systems, Market 


\section{Introduction}

DRC post-conflict situation is characterized by lack of food supply and proteins, especially animal proteins. Instead the end of the wars called African world war in July 1999 with Lusaka peace agreement signed on one site between DRC government and his allies at another site with some East-Africans neighbor's countries, until nowadays troubles persist in the eastern part of the country. Livestock decreased dramatically due to looting during the wars in 1996 à 2009 and in the troubles. This diminution is also due to low diseases control, lack of animal nutrition and extension services. As observed [1], the animal-agriculture production is not enough and livestock production is maintaining in extensive system. The yield crops are also low due to lack of good soil management, small lands of farmers especially in eastern part of the country. Population malnutrition rate is $15 \%$ [2]. Then, importation of meat is very high. In 2010, DRC imported 2853 tons of cattle meat, 59,468 tons of chickens, 132,915 tons of frozen fish [3]. However, the current President of DRC decided in 2019 to improve agriculture as one of major motors of the national development. In this way, promotion in the country of poultry industry should boost easily the animal protein's production and income generation due to their short breeding cycle. The poultry and their products are used for home consumption, as gifts, or for religious purposes. Additionally, they are sold to earn some income... Poultry are relatively cheap than the big animals, easy to rear, and easy to manage. Consequently, there has been and there is a growing attention and interest in poultry production in villages as well as in peri-urban and urban areas throughout the developing world [4]. The objective of this survey was to explore the chicken production systems and the constraints related in the way to set up a good chicken industry in the country in the coming peace period.

\section{Material and Methods}

\subsection{Sites Location}

Located in Central Africa, the Democratic Republic of the Congo (DRC) has $2,345,409 \mathrm{~km}^{2}$ and is the second big country in Africa. His inhabitants are estimated at $71,712,867$ persons and almost $65 \%$ live in rural area. The year increment rate of population is $2.7 \%$. The contribution of agriculture on GDP was $43 \%$ in 2009 [5] and the contribution of livestock on agriculture is 9.2\% [6]. The surveyed sites were Kinshasa; located in West is the capital town (see Figure 1); Lubumbashi the second country town is located in Katanga province in the South-East, in Kongo central province (Mvuazi-Nkolo) in West near Kinshasa, Goma a principal town and Bweremana-Sake villages in Nord-Kivu province and Bukavu the principal town of Sud-Kivu province and his hinterland of around $50 \mathrm{~km}$ in Sud-Kivu province. The two last provinces are located in the East.

\subsection{Survey Establishment}

To carry out the survey, two questionnaires were submitted. 


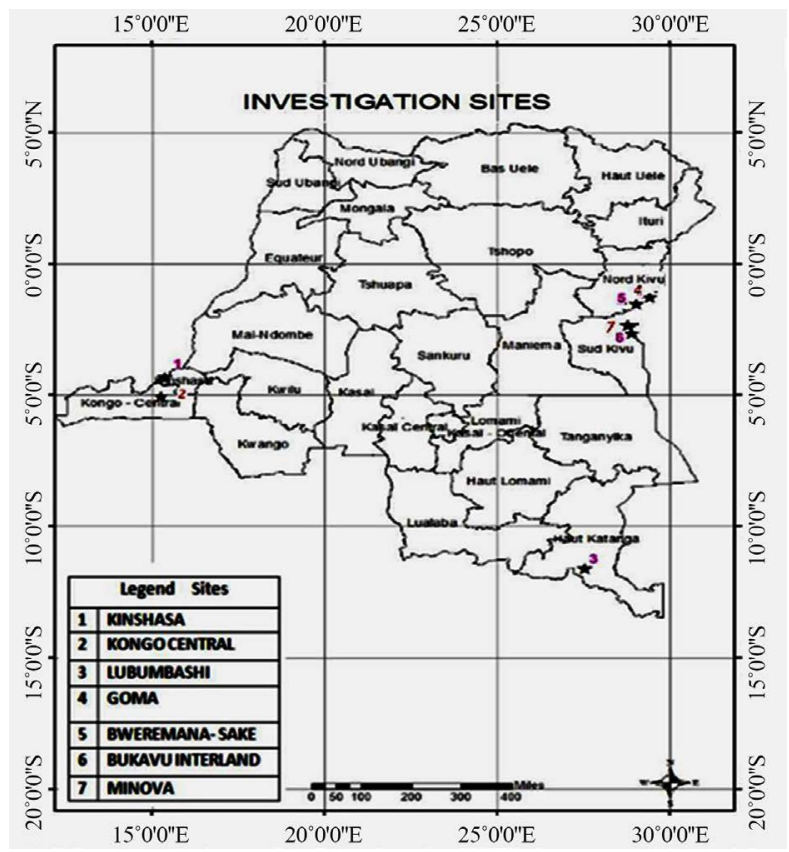

Figure 1. Localization of the study area.

\subsubsection{Farmer's Production Systems}

During the interview, seven sites described above and sampling were chosen randomly in the countries. Surveys were implemented from 8th to 23rd March 2015. We got at all 245 samples in which 170 samples from farmer's production systems and 75 samples from chickens marketing. About the farmer's production systems, survey was conducted at the household and chicken company level in the sites using standard methods of household interviews and structured questionnaires. About typology on farmer's productions, the number of chickens per farmer was: the big farmer more than 100, the middle farmer between 50 to 100 and the small farmers between 2 to 49 . Sample's number per site was 3 households for the big farmers, 7 households for the middle farmers and 15 households for the small farmers. Data were collected on the socio demographic characteristics, on production systems adopted by farmers, type of breeds of chicken reared, sources of stock, flock size and composition, management and housing systems adopted, feeding systems, diseases and production poultry constraints.

\subsubsection{Chicken's Commercialization}

Samples per site were 8 small traders, 2 wholesalers and 3 restaurants. Prices purchases and sold were collected to get a benefit margin. Information on improved chicken breed, country origin, transport and chicken meat processing, chicken products transportation were collected from the farmers. The number of animals was converted into Tropical Livestock Unit (TLU), where cattle are weighed with 0.7 , sheep/goat with 0.1 , swine 0.2 , and chicken 0.01 [7], rabbit 0.01 TLU, cavy 0.005 TLU [8]. Data were analyzed using descriptive analysis such as frequency distribution, percentages and means comparison. Statistical analyses 
were done in IBM SPSS Statistics version 20 software.

\section{Results and Discussion}

\subsection{Farmer's Production Systems}

\subsubsection{Social Characterization of Chicken's Farmers}

Results on Table 1 show that the chicken enterprise of the poultry sub-sector in DRC had a gender dominated by male farmers across the locations with participation level of $63.7 \%$ while female farmers constituted $36.3 \%$. In Sud-Kivu, DRC [9] observed that $66.6 \%$ of men are chicken farmers. In addition, the repartition of the average age of the chicken's farmers is 44.9 years. They were significant ( $\mathrm{P}$ $<0.05)$ differences in the average ages across the sites. It shows that farmers still are strong to rear the chickens; the youngest had 17 years old even if the oldest was 82 years old. According to the education level of the farmers, majority of them did the secondary school $48.7 \% \%$ follow by university $28.9 \%$ and primary school $15.1 \%$. Few interviewees were illiterates $7.3 \%$. Many famers reached the secondary school and university level as observed [10] in Sud-Kivu. The average experience of chicken's rearing per group age is recorded up to five years to $3.0 \pm$ 0.6 years, the group age 6 to 10 years of chicken experience had $8.0 \pm 1.7$ years and the group above 10 years had $20.1 \pm 8.2$ old. About interviewer's position, they were especially dominated by the responsible of household or enterprise at $63.5 \%$, the household children $24.1 \%$, agents represented $7.9 \%$ and spouse $4.3 \%$.

\subsubsection{Livestock Production in the Study Area}

Table 2 shows that the main TLU number of animals was recorded on cattle $14.5 \pm 40.0$ TLU followed by the chickens $4.5 \pm 40.0 \mathrm{TLU}$, swine $4.4 \pm 83 \mathrm{TLU}$ with high significant $(\mathrm{P}<0.001)$ differences between TLU swine averages across the sites.

The high record averages were observed on cattle with $28.5 \pm 23.2$ TLU in Lubumbashi, swine 19.6 \pm 26.6 TLU in Bukavu and his hinterland, sheep $2.4 \pm$ 2.9 TLU in Bweremana-Sake 8.3 TLU, goat 4.0 TLU in Lubumbashi, rabbit $0.9 \pm$ 1.0 in Kinshasa and Cavy in Bukavu and his hinterland.

\subsubsection{Chicken's Production Systems}

Chickens types reared in DRC, see Table 3, were commonly represented by the local one with $82.4 \%$ and the improved chicken at $17.6 \%$. Chickens in Sud-Kivu are mostly rearing in free-range system [10]. Among the improved chickens, Leghorn is first with $29.9 \%$, Derco ponte $22.2 \%$, Sussex hermine $15.5 \%$ and Rodes Island Red 13.4\%. According to the composition of chicken's flock size, it appeared that at the basic level, the rate of chicks was very important $60.5 \%$ but unfortunately from this stage to the mature birds, it decreased very drastically with successively; pullet $10.5 \%$, cockerel $8.0 \%$, cock $10.6 \%$ and hen $10.6 \%$. [9] observed that the rate of chickens at weaning period was $57.25 \%$ in Sud-Kivu. Chicks were not in generally cured or benefit of diseases prevention and any protection against bad weather or rapt by predators was done. If these gaps 
should be avoided, farmer can improve the chicken production from 30 up to $60 \%$. About the chicken house, the common one is the kitchen and free range $38.7 \%$ followed by chickens accommodated in human house $23.7 \%$, kitchen with enclosure $19.2 \%$ and chicken house built with bricks $18.6 \%$. On-farm, majority of chicken's producers $93.9 \%$ doesn't have hatchery, only $6.1 \%$ used it. Only hens play the role of incubating eggs. In Uganda, [11] observed that the use of hatchery is low due to lack knowledge of farmers in management, lack of fund and small chicks supply from parental strains.

Table 1. Farmer's social characterization.

\begin{tabular}{|c|c|c|c|c|c|c|c|c|c|}
\hline $\begin{array}{l}\text { Characteristics of } \\
\text { Chicken's Farmers }\end{array}$ & $\begin{array}{l}\text { Bukavu and } \\
\text { Hinterland }\end{array}$ & $\begin{array}{c}\text { Bweremana- } \\
\text { Sake }\end{array}$ & Goma & Kinshasa & $\begin{array}{l}\text { Kongo } \\
\text { Central }\end{array}$ & Lubumbashi & Minova & Total & $\begin{array}{l}\text { Test Stat. } \\
\text { Value (F) }\end{array}$ \\
\hline \multicolumn{10}{|l|}{ Sex } \\
\hline Male & $20(80)$ & $17(68)$ & $11(44)$ & $13(54.2)$ & $16(64)$ & $12(60)$ & $19(76)$ & $108(63.9)$ & \\
\hline Female & $5(20)$ & $8(32)$ & $14(56)$ & $11(45.8)$ & $9(36)$ & $8(40)$ & $6(24)$ & $61(36.1)$ & \\
\hline \multicolumn{10}{|l|}{$\begin{array}{l}\text { Age of } \\
\text { farmers }\end{array}$} \\
\hline Minimum age & 20 & 28 & 17 & 29 & 21 & 30 & 20 & 165 & \\
\hline Maximum age & 70 & 82 & 70 & 75 & 59 & 73 & 59 & 488 & \\
\hline Average age & $45.4 \pm 13.6$ & $46.2 \pm 12.4$ & $43.4 \pm 13.5$ & $48.9 \pm 11.2$ & $41.8 \pm 10.9$ & $51.3 \pm 10.4$ & $38.2 \pm 11.9$ & $44.9 \pm 12.6$ & $2.829^{* *}$ \\
\hline \multicolumn{10}{|l|}{ Education } \\
\hline Illiterate & $1(4.2)$ & $2(9.5)$ & $1(4.5)$ & & $2(10)$ & $1(5.6)$ & $4(16)$ & $11(7.3)$ & \\
\hline Primary & $6(25)$ & $5(23.8)$ & $2(9.1)$ & $3(13.6)$ & $1(5)$ & $2(11.1)$ & $4(16)$ & $23(15.1)$ & \\
\hline Secondary & $13(54.2)$ & $12(57.1)$ & $10(45.5)$ & $10(45.5)$ & $10(50)$ & $6(33.3)$ & $13(52)$ & $74(48.7)$ & \\
\hline University & $4(16.7)$ & $2(9.5)$ & $9(40.9)$ & $9(40.9)$ & $7(35)$ & $9(50)$ & $4(16)$ & $44(28.9)$ & \\
\hline \multicolumn{10}{|l|}{$\begin{array}{c}\text { Years' } \\
\text { experience }\end{array}$} \\
\hline Up to 5 years & $2.9 \pm 1.7$ & $3.4 \pm 1.4$ & $2.1 \pm 1.1$ & $4.0 \pm 1.3$ & $2.9 \pm 1.4$ & $2.0 \pm 1.8$ & $3.6 \pm 1.2$ & $3.0 \pm 0.6$ & \\
\hline $6-10$ years & $8.2 \pm 2.0$ & $7.5 \pm 2.0$ & $8.0 \pm 2.0$ & $8.4 \pm 1.7$ & $8.4 \pm 1.7$ & $7.6 \pm 1.7$ & $9.0 \pm 1.7$ & $8.0 \pm 1.7$ & \\
\hline Above 10 years & $17.8 \pm 5.7$ & $23.1 \pm 7.7$ & $23.8 \pm 13.3$ & $20.5 \pm 8.3$ & $17.0 \pm 4.1$ & $16.0 \pm 4.2$ & $25.3 \pm 10.5$ & $20.1 \pm 8.2$ & \\
\hline \multicolumn{10}{|l|}{$\begin{array}{l}\text { Position of the } \\
\text { responding }\end{array}$} \\
\hline $\begin{array}{c}\text { Responsible of } \\
\text { Household } \\
\text { /enterprise }\end{array}$ & $21(84)$ & $14(56)$ & $20(80)$ & $10(40)$ & $14(56)$ & $10(50)$ & $20(80)$ & $109(66.5)$ & \\
\hline Children & & $9(36)$ & $5(20)$ & $12(48)$ & $7(28)$ & $5(25)$ & $3(12)$ & $35(21.3)$ & \\
\hline Agent & $2(8)$ & & & $3(12)$ & $4(16)$ & $4(20)$ & & $13(7.9)$ & \\
\hline Spouse & $2(8)$ & $2(8)$ & & & & $1(5)$ & $2(8)$ & $7(4.3)$ & \\
\hline
\end{tabular}

** Significant at $\mathrm{P}<0.05$. 
Table 2. Animal species reared (Averages in TLU).

\begin{tabular}{|c|c|c|c|c|c|c|c|c|c|}
\hline $\begin{array}{l}\text { Animal Species per } \\
\text { Household }\end{array}$ & $\begin{array}{l}\text { Bukavu and } \\
\text { Hinterland }\end{array}$ & $\begin{array}{l}\text { Bweremana- } \\
\text { Sake }\end{array}$ & Goma & Kinshasa & $\begin{array}{l}\text { Kongo } \\
\text { Central }\end{array}$ & Lubumbashi & Minova & Total & $\begin{array}{l}\text { Test Stat. } \\
\text { Value (F) }\end{array}$ \\
\hline Cattle & & & $5.9 \pm 6.4$ & 1.4 & & $28.5 \pm 23.2$ & 2.8 & $14.5 \pm 40.0$ & \\
\hline Chickens & $0.5 \pm 0.7$ & $0.4 \pm 0.8$ & $0.4 \pm 0.4$ & $5.1 \pm 15.9$ & $0.7 \pm 1.0$ & $29.0 \pm 114.4$ & $0.5 \pm 0.5$ & $4.5 \pm 40.0$ & 0.941 \\
\hline Swine & $19.6 \pm 26.6$ & $0.4 \pm 0.1$ & $1.8 \pm 1.0$ & $5.6 \pm 3.2$ & $2.3 \pm 3.0$ & $10.1 \pm 8.2$ & $0.8 \pm 0.9$ & $4.4 \pm 8.3$ & $4.101^{* * *}$ \\
\hline Sheep & & 8.3 & & 1.5 & $0.9 \pm 0.8$ & $1.7 \pm 1.9$ & 1.3 & $2.4 \pm 2.9$ & \\
\hline Goat & $0.3 \pm 0.2$ & 0.4 & $0.5 \pm 0.3$ & & & 4.0 & $0.5 \pm 0.5$ & $0.6 \pm 0.9$ & \\
\hline Rabbit & & $0.3 \pm 0.2$ & $0.08 \pm 0.1$ & $0.9 \pm 1.0$ & $0.4 \pm 0.3$ & & 0.08 & $0.3 \pm 0.4$ & \\
\hline Cavy & $0.2 \pm 0.5$ & & & & 0.06 & & 0.04 & $0.1 \pm 0.7$ & \\
\hline
\end{tabular}

*** Significant at $\mathrm{P}<0.001$.

Table 3. Chicken types and flock organization.

\begin{tabular}{|c|c|c|c|c|c|c|c|c|}
\hline $\begin{array}{l}\text { Characteristics } \\
\text { per Household }\end{array}$ & $\begin{array}{l}\text { Bukavu and } \\
\text { Hinterland }\end{array}$ & $\begin{array}{l}\text { Bweremana- } \\
\text { Sake }\end{array}$ & Goma & Kinshasa & $\begin{array}{l}\text { Kongo } \\
\text { Central }\end{array}$ & Lubumbashi & Minova & Total \\
\hline \multicolumn{9}{|l|}{ Chicken's types reared } \\
\hline Local & $10(71.4)$ & $18(78.3)$ & $11(85.6)$ & $15(62.5)$ & $7(100.0)$ & $20(90.9)$ & $6(88.9)$ & $89(82.4)$ \\
\hline Improved & $4(28.6)$ & $5(21.7)$ & $2(15.4)$ & $9(37.5)$ & & $2(9.1)$ & $1(11.1)$ & $23(17.6)$ \\
\hline Leghorn & & $9(60.0)$ & $1(10.0)$ & $2(16.7)$ & $3(25.0)$ & $3(33.3)$ & $9(64.3)$ & $27(29.9)$ \\
\hline Sussex hermine & $2(25.0)$ & & $2(20.0)$ & $3(25.0)$ & $2(16.7)$ & $2(22.2)$ & & $11(15.5)$ \\
\hline Derco ponte & $4(50.0)$ & $4(26.7)$ & $2(20.0)$ & $1(8.3)$ & $3(25.0)$ & $1(11.1)$ & $2(14.3)$ & $17(22.2)$ \\
\hline Rhodes Island Red & $1(12.5)$ & $1(6.7)$ & $2(20.0)$ & $1(8.3)$ & $2(16.7)$ & $2(22.2)$ & $1(7.1)$ & $10(13.4)$ \\
\hline Arbor & & $1(6.7)$ & $1(10.0)$ & $4(33.3)$ & $1(8.3)$ & & & $9(10.4)$ \\
\hline Unknown & $1(12.5)$ & & $2(20.0)$ & $1(8.3)$ & $1(8.3)$ & $1(11.1)$ & & $6(8.6)$ \\
\hline \multicolumn{9}{|l|}{ Composition of chicken' } \\
\hline \multicolumn{9}{|l|}{ flocks size } \\
\hline Chicks & $19(65.5)$ & $18(75.0)$ & $20(74.1)$ & $17(56.7)$ & $4(25.0)$ & $14(66.7)$ & $15(60)$ & $107(60.5)$ \\
\hline Pullet & $2(6.9)$ & $1(4.2)$ & $1(3.7)$ & $3(10.0)$ & $4(25.0)$ & $3(14.3)$ & $2(8)$ & $16(10.3)$ \\
\hline Cockerel & $4(13.8)$ & $1(4.2)$ & $2(7.4)$ & $1(3.3)$ & $3(18.8)$ & $1(4.8)$ & $1(4)$ & $13(8.0)$ \\
\hline Hen & $2(6.9)$ & $2(8.3)$ & $3(11.1)$ & $5(16.7)$ & $3(18.8)$ & $1(4.8)$ & $2(8)$ & $18(10.6)$ \\
\hline Cock & $2(6.9)$ & $2(8.3)$ & $1(3.7)$ & $4(13.3)$ & $2(12.5)$ & $2(9.5)$ & $5(20)$ & $18(10.6)$ \\
\hline \multicolumn{9}{|l|}{ Chickens' houses } \\
\hline Chickens in human house & $6(27.3)$ & $6(28.6)$ & $9(37.5)$ & $3(12)$ & $2(10)$ & $7(35)$ & $4(16.7)$ & $37(23.7)$ \\
\hline Kitchen and enclosure & $3(13.6)$ & $3(14.3)$ & $2(8.3)$ & $4(16)$ & $3(15)$ & $8(40)$ & $7(29.2)$ & $30(19.2)$ \\
\hline House built with bricks & $6(27.3)$ & $4(19.0)$ & $1(4.2)$ & $13(52)$ & $1(5)$ & $2(10)$ & $28.3)$ & $29(18.6)$ \\
\hline \multicolumn{9}{|l|}{ Presence or not of hatchery } \\
\hline Yes, I have & & $1(14.3)$ & & & $2(28.6)$ & & & $3(6.1)$ \\
\hline No, I don't have & $24(100.0)$ & $6(85.7)$ & $2(100.0)$ & $19(86.4)$ & $5(71.4)$ & $13(100.0)$ & $11(100.0)$ & $78(93.9)$ \\
\hline
\end{tabular}

Values in parentheses are percentages. 


\subsubsection{Chicken's Health and Nutrition}

Among the major diseases (see Table 4), New Castle was recorded first $68.4 \%$, followed by Gumboro 16.9\%, Marek and Salmonellosis 5.4\% diseases. The total average of death rate was $23.5 \% \pm 26.0 \%$ with high significant $(\mathrm{P}<0.001)$ differences in the average of global mortality rates across the sites. According to the seasons per year, the wet season is very hard with $42.7 \%$ of death in terms of incidence of diseases attack on-farm level. In dry season, it represented $33.7 \%$ and both the two seasons $23.6 \%$. Mortality rate is mostly so high.

Table 4. Health and nutrition management.

\begin{tabular}{|c|c|c|c|c|c|c|c|c|c|}
\hline Characteristics & $\begin{array}{c}\text { Bukavu and } \\
\text { Hinterland }\end{array}$ & $\begin{array}{c}\text { Bweremana- } \\
\text { Sake }\end{array}$ & Goma & Kinshasa & $\begin{array}{l}\text { Kongo } \\
\text { Central }\end{array}$ & Lubumbashi & Minova & Total & $\begin{array}{l}\text { Test Stat. } \\
\text { Value (F) }\end{array}$ \\
\hline \multicolumn{10}{|l|}{ Important diseases } \\
\hline New Castle & $14(82.4)$ & $8(40.0)$ & $19(100)$ & $9(69.2)$ & $5(83.3)$ & $2(50.0)$ & $7(53.8)$ & $64(68.4)$ & \\
\hline Gumboro & & $3(15.0)$ & & $4(30.8)$ & $1(16.7)$ & $1(25.0)$ & $4(30.8)$ & $13(16.9)$ & \\
\hline Marek & & $1(5.0)$ & & & & $1(25.0)$ & $1(7.7)$ & $3(5.4)$ & \\
\hline Parasites & & $1(5.0)$ & & & & & & $1(0.7)$ & \\
\hline Salmonellosis & $2(11.8)$ & $3(15.0)$ & & & & & $1(7.7)$ & $6(4.9)$ & \\
\hline Bronchitis infectious & & $1(5.0)$ & & & & & & $1(0.7)$ & \\
\hline Diphtheria & & $1(5.0)$ & & & & & & $1(0.7)$ & \\
\hline Coccidiosis & & $2(10.0)$ & & & & & & $3(5.4)$ & \\
\hline \multicolumn{10}{|l|}{$\begin{array}{l}\text { Seasonal frequency } \\
\text { of diseases }\end{array}$} \\
\hline Wet season & $1(16.7)$ & $11(57.9)$ & $8(34.8)$ & $2(18.2)$ & $2(33.3)$ & $1(16.7)$ & $13(72.2)$ & $38(42.7)$ & \\
\hline Dry season & $2(33.3)$ & $6(31.6)$ & $6(26.1)$ & $7(63.6)$ & $4(66.7)$ & $2(33.3)$ & $3(16.7)$ & $30(33.7)$ & \\
\hline Both seasons & $3(50.0)$ & $2(10.5)$ & $9(39.1)$ & $2(18.2)$ & & $3(50)$ & $2911.1)$ & $21(23.6)$ & \\
\hline Global rate of death & $14 \pm 12.6$ & $46.9 \pm 26.0$ & $23.7 \pm 25.0$ & $9.1 \pm 10.2$ & $5.2 \pm 2.9$ & $9.0 \pm 6.4$ & $53.4 \pm 35.7$ & $23.5 \pm 26.0$ & $1.25^{\star * *}$ \\
\hline \multicolumn{10}{|l|}{ Using veterinary services } \\
\hline Yes, I use it & $3(25)$ & $3(15.0)$ & $6(35.3)$ & $7(58.3)$ & $3(23.1)$ & $8(80.0)$ & $2(14.3)$ & $32(35.9)$ & \\
\hline No, I don't use it & $9(75)$ & $17(85.0)$ & $11(64.7)$ & $5(41.7)$ & $10(76.9)$ & $2(20.0)$ & $12(85.7)$ & $66(64.1)$ & \\
\hline \multicolumn{10}{|l|}{ Nutrition systems } \\
\hline Scavenging & $1(4.3)$ & $3(16.7)$ & $2(8.3)$ & $3(12.5)$ & $6(26.1)$ & & $6(25.0)$ & $21(13.3)$ & \\
\hline $\begin{array}{l}\text { Scavenging/grains/ } \\
\text { kitchen residues }\end{array}$ & $10(43.5)$ & $6(33.3)$ & $10(41.7)$ & $11(45.8)$ & $11(47.8)$ & $2(10.5)$ & $7(29.2)$ & $57(36.0)$ & \\
\hline $\begin{array}{l}\text { Concentrate with } \\
\text { scavenging }\end{array}$ & $9(39.1)$ & $6(33.3)$ & $3(12.5)$ & $3(12.5)$ & $3(13.0)$ & $1(5.3)$ & $4(16.7)$ & $32(30.0)$ & \\
\hline Concentrate & $3(13.0)$ & $3(16.7)$ & $9(37.5)$ & $(729.2)$ & $3(13.0)$ & $16(84.2)$ & $7(29.2)$ & $45(20.7)$ & \\
\hline \multicolumn{10}{|l|}{ On-farm feed distribution } \\
\hline Parents & $9(39.1)$ & $15(71.4)$ & $9(36.0)$ & $14(56.0)$ & $12(60.0)$ & $5(26.3)$ & $19(79.2)$ & $83(52.6)$ & \\
\hline Family members & $9(39.1)$ & $5(23.8)$ & $7(28.0)$ & & $2(10.0)$ & $3(15.8)$ & $1(4.2)$ & $27(17.3)$ & \\
\hline Children & $4(17.4)$ & & $3(12.0)$ & $4(16.0)$ & $5(25.0)$ & $7(36.8)$ & $2(8.3)$ & $25(16.5)$ & \\
\hline Agents & $1(4.3)$ & $1(4.8)$ & $6(24.0)$ & $7(28.0)$ & $1(5.0)$ & $4(21.1)$ & $2(8.3)$ & $22(13.6)$ & \\
\hline
\end{tabular}

${ }^{* * *}$ Significant at $\mathrm{P}<0.001$, values in parentheses are percentages. 
Through the sites, the rate of death was high as recorded in Minova 53.4\% \pm $35.7 \%$ and Bweremana-Sake $46.9 \% \pm 26.0 \%$. In Uganda, [12] spoke about diseases of high importance which always cause high mortality (kill over $50 \%-90 \%$ of the stock) and are difficult to treat. In Cameroon, mortality caused by diseases can sometimes attend $100 \%$ [13]. On chicken feeds, the most nutrition system is in scavenging. The personnel who nourish the chickens is mostly represented by the parents within the family $52.6 \%$ followed by all the family members $17.3 \%$, children $16.5 \%$ and agents $13.6 \%$ from enterprises and households.

The support of extension services was almost inexistent in the chicken's production systems, $78.2 \%$ of interviewees were not assisted, see Table 5 . The one who assisted few farmers were represented by NGO's at $58.1 \%$, private sector $27.9 \%$ and the last one was the public sector $14.1 \%$ which was represented mostly by the agricultural scientist in scaling up on some livestock topics. In Colombia, [14] declared that regarding to the training/extension service situation $45 \%$ of the interviewed smallholder producers had received formal training (held by a Non-Governmental Organization (NGO), a Governmental Organization (GO), or by a feed supplier). The distance from farm-gate to veterinary pharmacy is high in big towns Kinshasa and Lubumbashi than in middle towns and villages, due probably to size of agglomeration.

\subsection{Chicken's Commercialization}

\subsubsection{Chicken Commercialization and Social Characterization of the Chicken's Traders}

Majority of chicken's traders were male $61.3 \%$, see Table 6 . Regarding to their education, the secondary school $56.3 \%$ prevailed followed by primary school $19.7 \%$, university $16.9 \%$ and illiterates $7.1 \%$. The trader's experience of the first group age below to 5 years had an average of $3.0 \pm 1.3$ years old. The second group age 6 to 10 years had an average of $7.6 \pm 1.8$ years, up to 10 years' group, $21.2 \pm 9.4$ years old. The average age was $37.9 \pm 10.3$ years with high significant $(\mathrm{P}<0.001)$ differences across the sites.

\subsubsection{Chicken Marketing Channel}

With regard to Table 7, chicken meat was almost not transformed, 94.8\%. The chicken prices were for the cock $12.0 \pm 2.6 \$$, hen $5.9 \pm 1.4 \$$ with significant $(\mathrm{P}<$ $0.05)$ differences in average hen prices across the sites and egg $0.24 \pm 0.39 \$$ with high significant $(\mathrm{P}<0.001)$ differences in the average egg prices across the sites. In Senegal at farm-gate, price of one chicken kilogram in 2011 was 2.1 USD $\$$ and at consumer level was 3.6 USD \$ [15]. As chicken price is very high in DRC, it is a good opportunity for the producers who would like to intensify the chicken industry production because market is available. The common transport of chickens, food, eggs and materials was especially done on foot $36.4 \%$ followed by car $31.8 \%$ and motorcycle $17.1 \%$. The imported chickens were principally coming from Belgium and Rwanda. Among the types of chickens imported were manly broilers $77.5 \%$ and spent layers $22.5 \%$. 
Table 5. Extension and private services.

\begin{tabular}{|c|c|c|c|c|c|c|c|c|}
\hline Characteristics & $\begin{array}{c}\text { Bukavu } \\
\text { and } \\
\text { Hinterland }\end{array}$ & $\begin{array}{c}\text { Bweremana- } \\
\text { Sake }\end{array}$ & Goma & Kinshasa & $\begin{array}{l}\text { Kongo } \\
\text { Central }\end{array}$ & Lubumbashi & Minova & Total \\
\hline \multicolumn{9}{|l|}{$\begin{array}{c}\text { Support } \\
\text { of extension } \\
\text { service }\end{array}$} \\
\hline Yes & $2(9.5)$ & $1(10.0)$ & $3(13.6)$ & $3(17.6)$ & $5(83.3)$ & $4(80.0)$ & $1(16.7)$ & $19(21.9)$ \\
\hline No & $19(90.5)$ & $9(90.0)$ & $19(86.4)$ & $14(82.4)$ & $1(16.7)$ & $1(20.0)$ & $5(83.3)$ & $68(78.2)$ \\
\hline \multicolumn{9}{|l|}{$\begin{array}{l}\text { Extension } \\
\text { service } \\
\text { activities }\end{array}$} \\
\hline Public sector & $1(11.1)$ & & $1(16.7)$ & $1(25.0)$ & $1(25.0)$ & $1(14,3)$ & $1(11.1)$ & $6(14.0)$ \\
\hline NGO & $6(66.7)$ & $3(100.0)$ & $3(50.0)$ & $2(50.0)$ & $2(50.0)$ & $3(42.9)$ & $6(66.7)$ & $25(58.1)$ \\
\hline Private sector & $2(22.2)$ & & $2(33.3)$ & $1(25.0)$ & $1(25,0)$ & $3(42,9)$ & $2(22.2)$ & $12(27.9)$ \\
\hline $\begin{array}{l}\text { Pharmacy } \\
\text { and farmer's } \\
\text { gate distance }\end{array}$ & $3.4 \pm 7.5$ & $1.7 \pm 2.3$ & $1.5 \pm 1.4$ & $4.4 \pm 4.3$ & & $5,9 \pm 10.8$ & $2.2 \pm 2.2$ & $3.0 \pm 5.0$ \\
\hline
\end{tabular}

Values in parentheses are percentages.

Table 6. Social characterization of chicken's traders.

\begin{tabular}{|c|c|c|c|c|c|c|c|c|c|}
\hline $\begin{array}{c}\text { Social } \\
\text { Characteristics }\end{array}$ & $\begin{array}{c}\text { Bukavu } \\
\text { and } \\
\text { Hinterland }\end{array}$ & $\begin{array}{c}\text { Bweremana- } \\
\text { Sake }\end{array}$ & Goma & Kinshasa & $\begin{array}{l}\text { Kongo } \\
\text { Central }\end{array}$ & Lubumbashi & Minova & Total & $\begin{array}{l}\text { Test Stat. } \\
\text { Value (F) }\end{array}$ \\
\hline \multicolumn{10}{|l|}{ Sex } \\
\hline Male & $10(76.9)$ & $5(71.4)$ & $6(42.9)$ & $4(28.6)$ & $6(100.0)$ & $5(71.4)$ & $10(71.4)$ & $46(61.3)$ & \\
\hline Female & $10(23.1)$ & $2(28.6)$ & $8(57.1)$ & $10(71.4)$ & & $2(28.6)$ & $4(28.6)$ & $29(38.7)$ & \\
\hline \multicolumn{10}{|l|}{ Education } \\
\hline Illiterate & $2(18.3)$ & & $1(7.1)$ & & & & $2(16.7)$ & $5(7.1)$ & \\
\hline Primary & $5(45.5)$ & $1(14.3)$ & $4(28.6)$ & $3(21.4)$ & & $2(28.6)$ & $1(8.3)$ & $14(19.7)$ & \\
\hline Secondary & $4(36.4)$ & $6(85.7)$ & $5(35.75)$ & $9(64.3)$ & $6(100.0)$ & $5(71.4)$ & $8(66.7)$ & $40(56.3)$ & \\
\hline University & & $6(33.3)$ & $4(28.5)$ & $2(14.3)$ & & $1(5.3)$ & $1(8.3)$ & $12(16.9)$ & \\
\hline \multicolumn{10}{|l|}{$\begin{array}{c}\text { Years' } \\
\text { experience }\end{array}$} \\
\hline Below 5 years & $2.8 \pm 1.6$ & $2.7 \pm 1.0$ & $2.5 .5 \pm 1.9$ & & $2.7 \pm 0.9$ & 3.0 & $3.5 \pm 1.2$ & $3.0 \pm 1.3$ & \\
\hline 6 to 10 years & 7.0 & 7.0 & & $7.4 \pm 1.7$ & 10.0 & $8.0 \pm 2.8$ & $7.3 \pm 2.3$ & $7.6 \pm 1.8$ & \\
\hline Up to 10 years & $17.5 \pm 4.8$ & $13.5 \pm 2.1$ & $27.8 \pm 12.2$ & $22.0 \pm 6.1$ & & $17.5 \pm 3.5$ & $16.0 \pm 3.6$ & $21.2 \pm 9.4$ & \\
\hline $\begin{array}{l}\text { Average age } \\
\text { of traders }\end{array}$ & $36.8 \pm 5.4$ & $34.0 \pm 6.8$ & $40.2 \pm 13.9$ & $42.9 \pm 7.3$ & $35.2 \pm 5.4$ & $47.4 \pm 10.3$ & $29.7 \pm 8.0$ & $37.9 \pm 10.3$ & $4341^{\star * *}$ \\
\hline
\end{tabular}

*** Significant at $\mathrm{P}<0.001$, values in parentheses are percentages. 
Table 7. Marketing channel and price for chicken products.

\begin{tabular}{|c|c|c|c|c|c|c|c|c|c|}
\hline Characteristics & $\begin{array}{l}\text { Bukavu and } \\
\text { Hinterland }\end{array}$ & $\begin{array}{l}\text { Bweremana- } \\
\text { Sake }\end{array}$ & Goma & Kinshasa & $\begin{array}{l}\text { Kongo } \\
\text { Central }\end{array}$ & Lubumbashi & Minova & Total & $\begin{array}{l}\text { Test Stat. } \\
\text { Value (F) }\end{array}$ \\
\hline \multicolumn{10}{|l|}{ Chicken's processing } \\
\hline No & $10(100.0)$ & $2(100.0)$ & $10(100.0)$ & $5(83.3)$ & $1(100.0)$ & $4(80.0)$ & $5(100.0)$ & $37(94.8)$ & \\
\hline Yes & & & & $1(16.6)$ & & $1(20.0)$ & & $2(5.2)$ & \\
\hline \multicolumn{10}{|l|}{$\begin{array}{l}\text { Sold price of local } \\
\text { chickens (\$) }\end{array}$} \\
\hline Cock & $12.7 \pm 3.5$ & $12.4 \pm 2.9$ & $13.7 \pm 2.5$ & & & $11.3 \pm 1.3$ & $10.5 \pm 2.3$ & $12.0 \pm 2.6$ & \\
\hline Hen & $6.8 \pm 1.1$ & $5.5 \pm 1.6$ & 7.0 & $5.5 \pm 1.1$ & $3.7 \pm 0.3$ & 5.2 & $6.7 \pm 1.1$ & $5.9 \pm 1.4$ & $2.688^{*}$ \\
\hline Egg & $0.15 \pm 0.9$ & $0.19 \pm 0.1$ & $0.19 \pm 0.3$ & $0.15 \pm 0.1$ & $0.17 \pm 0.1$ & $0.39 \pm 0.2$ & $0.18 \pm 0.2$ & $0.24 \pm 0.4$ & $7.789^{* * *}$ \\
\hline \multicolumn{10}{|l|}{$\begin{array}{l}\text { Chickens transport } \\
\text { and supplies }\end{array}$} \\
\hline Foot & $21(55)$ & $11(55)$ & & $18(72.0)$ & $7(33.3)$ & & $1(7.1)$ & $58(36.4)$ & \\
\hline Lorry & $2(8.3)$ & $2(10.0)$ & & $3(12.0)$ & $12(57.1)$ & $7(70.0)$ & $9(64.3)$ & $35(31.8)$ & \\
\hline Motorcycle & $1(4.2)$ & $6(30)$ & $8(36.4)$ & $2(8.0)$ & $2(9.5)$ & $1(30.0)$ & $3(21.4)$ & $23(17.1)$ & \\
\hline Train & & & $11(50.0)$ & & & & & $11(7.1)$ & \\
\hline Bicycle & & $1(5.0)$ & $2(9.1)$ & $2(8.0)$ & & $2(20.0)$ & $1(7.1)$ & $8(7.0)$ & \\
\hline Mail & & & $1(4.5)$ & & & & & $1(0.6)$ & \\
\hline \multicolumn{10}{|l|}{$\begin{array}{l}\text { Country origin of } \\
\text { importation }\end{array}$} \\
\hline Belgium & $1(12.5)$ & & $1(8.3)$ & $7(100.0)$ & $1(100.0)$ & $1(16.7)$ & $1(20.0)$ & $12(27.3)$ & \\
\hline Rwanda & $5(62.5)$ & $3(60.0)$ & $2(16.7)$ & & & & $2(40.0)$ & $12(27.3)$ & \\
\hline Burundi & $2(25.0)$ & & $5(41.7)$ & & & & & 7 (15.9) & \\
\hline Kenya & & $1(20.0)$ & $4(33.3)$ & & & & $1(20.0)$ & $6(13.6)$ & \\
\hline Zambia & & & & & & $583.3)$ & & $5(11.4)$ & \\
\hline Uganda & & $1(20.0)$ & & & & & $1(20.0)$ & $2(4.3)$ & \\
\hline \multicolumn{10}{|l|}{$\begin{array}{l}\text { Chickens imported } \\
\text { breed types }\end{array}$} \\
\hline Broiler & $7(10.0)$ & $5(100.0)$ & $8(88.9)$ & $5(45.5)$ & $1(100.0)$ & $4(66.7)$ & $1(100.0)$ & $31(77.5)$ & \\
\hline Spent layers & & & $1(11.1)$ & $6(54.5)$ & & $2(33.3)$ & & $9(22.5)$ & \\
\hline
\end{tabular}

${ }^{*}$ Significant at $\mathrm{P}<0.05,{ }^{* *}$ Significant at $\mathrm{P}<0.001$, values in parentheses are percentages.

On Table 8, the brut margin benefit of eggs was $0.24 \pm 0.39 \$$ per egg with significant $(\mathrm{P}<0.05)$ differences of egg averages brut benefit margin across the sites and $3.36 \pm 4.18 \$$ per chicken with high significant $(\mathrm{P}<0.001)$ differences of brut benefit margin averages across the sites. In the restaurant, price is given for the entire menu and not only the chicken products. The brut margin benefit was calculated by purchase price to minus the sold one without considering the other 
expenses related. It seemed that chickens were very profitable than eggs. Management to sell many local chickens is difficult because people like to kill themselves the chickens, except meat from improved chickens sold in the towns.

\subsubsection{Weakness of Chicken's Production Systems}

Chickens production systems in DRC faced many problems that didn't allow them a good development of chicken industry. The first constraint declaimed by the interviewees was lack of credit 21.6. It was followed by the lack of feeds and medicines $19.6 \%$, lack of chicks supply $17.6 \%$. We noted that DRC didn't have a parental stock in all the country, mortality and morbidity caused by diseases represented $15.7 \%$ (Table 9).

The improved chickens are imported mainly from fertile eggs and chicks 1 day-old. To boost chicken's production, in Ethiopia, there are government-owned poultry breeding and rearing centers aimed at providing improved dual purpose chickens of exotic breeds [16]. All these results demonstrate the necessity to begin now the chicken industry program in DRC. It should start first by a local chicken's characterization coupled with genetic molecular analysis in the entire country before the selection begins. Local genetic materials should be conserved. At the same time, promotion of improved chickens with installing parental strains in at least five locations to cover all the country: Kinshasa, Lubumbashi, Kisangani, Bukavu and Bunia should be done. Roads should be good and agriculture improved for applying feeds from crops and processing residues. In Senegal, since 2005, importation of chicken meat was prohibited and then the national production increased [15].

Table 8. Profitability (\$) from chicken products en 2014.

\begin{tabular}{cccccc}
\hline Chechen Products & Trader & Wholesaler & Restaurant & Total & Test Stat. Value (F) \\
\hline Egg & $0.12 \pm 0.26$ & $0.20 \pm 0.31$ & $0.55 \pm 0.52$ & $0.24 \pm 0.39$ & $6.877^{* *}$ \\
Hen & $1.97 \pm 1.90$ & $3.87 \pm 4.40$ & $7.95 \pm 6.34$ & $3.36 \pm 4.18$ & $13.944^{* * *}$ \\
\hline
\end{tabular}

${ }^{* *}$ Significant at $\mathrm{P}<0.05 .{ }^{* *}$ Significant at $\mathrm{P}<0.001$.

Table 9. Technical constraints of chicken's production.

\begin{tabular}{|c|c|c|c|c|c|c|c|c|}
\hline Characteristics & $\begin{array}{l}\text { Bukavu and } \\
\text { Hinterland }\end{array}$ & Bweremana-Sake & Goma & Kinshasa & $\begin{array}{l}\text { Kongo } \\
\text { Central }\end{array}$ & Lubumbashi & Minova & Total \\
\hline Unavailability of credit & $1(16.7)$ & $2(25.0)$ & $3(37.5)$ & $1(16.7)$ & $1(16.7)$ & $1(16.7)$ & $2(18.1)$ & $11(21.6)$ \\
\hline Unavailability of medicines & $1(16.7)$ & $1(12.5)$ & $2(25.0)$ & $1(16.7)$ & $1(16.7)$ & $1(16.7)$ & $3(27.3)$ & $10(19.6)$ \\
\hline Unavailability of feeds & $1(16.7)$ & $1(12.5)$ & $2(25.0)$ & $1(16.7)$ & $1(16.7)$ & $1(16.7)$ & $3(27.3)$ & $10(19.6)$ \\
\hline Chicks supply & $1(16.7)$ & $2(25.0)$ & & $1(16.7)$ & $2(33.2)$ & $2(33.2)$ & $1(9.1)$ & $9(17.6)$ \\
\hline Disease's consequences & $2(33.2)$ & $1(12.5)$ & & $2(33.2)$ & $1(16.7)$ & $1(16.7)$ & $1(9.1)$ & $8(15.7)$ \\
\hline Market not organized & & $1(12.5)$ & $1(12.5)$ & & & & & $2(3.9)$ \\
\hline Inappropriate chicken's house & & & & & & & $1(9.1)$ & $1(2.0)$ \\
\hline
\end{tabular}

Values in parentheses are percentages. 


\section{Conclusion}

Chicken's technology is yet very low in the country; traditional system prevailed by high mortality, lack of animal feeds, inadequate accommodation... Improved chickens are not yet good scaling up and their one-day old chicks were rare. It was due especially to lack of parental stock strains in the country and a lack of local breeding program. The most constraints recorded were lack of credit, lack of medicines, feeds, one-day old chicks supply, high mortality and market access not yet well organized. The high prices of eggs and chickens should be very incentive to promote chicken's production in the country. Challenges enumerated above may be overcome. The high rate of malnutrition among the population should push the government to boost the chicken industry in the way to sustain poverty alleviation and to fight hunger. Government should then improve the local chickens and install some parental stock strains of improved chickens in the country. By the way, health control should be ensured and feeds been available and accessible with an intensification of agriculture and good roads to facilitate the market channel.

\section{Acknowledgements}

Research was funded by the Korea-Africa Food Agriculture Cooperation Initiative.

\section{Conflicts of Interest}

The authors declare no conflicts of interest regarding the publication of this paper.

\section{References}

[1] Dieudonné, K. (2014) Evaluation des légumineuses fourragères dans un système d'élevage Editions universitaires européennes.

http://www.editions-ue.com/

[2] SNSA, Service national des statistiques agricoles (2011) Service national des statistiques agricoles R. D. Congo.

[3] SNSA, Service national des statistiques agricoles mai (2012) L'agriculture congolaise en quelques chiffres Ministère national de l'agriculture et du développement rural Secrétariat général à l'agriculture, pêche et élevage.

[4] Permin, A. (2009) Good Practices in Small Scale Poultry Production: A Manual for Trainers and Producers in East Africa FAO.

[5] FAO, Food Agriculture Organization (2013) Democratic Republic of the Congo BEFS Country Brief.

[6] FAO, Food Agriculture Organization (2005) Livestock Brief, Congo, Democratic republic Livestock Information. Sector Analysis and Policy Branch AGAL, 15 p. http://www.fao.org/ag/againfo/resources/en/publications/sector_briefs/lsb_COD.pdf

[7] Ghirotti, M. (1993) Rapid Appraisal: Benefiting from the Experiences and Perspectives of Livestock Breeders. World Animal Review, 77, 26-37.

http://www.fao.org/docrep/V1650T/v1650T0d.htm 
[8] LEAD, Livestock, Environment and Development Initiative (1999) Tropical Livestock Units (TLU). In: Livestock \& Environment Toolbox, LEAD/FAO, Rome, Italy. http://www.fao.org/ag/againfo/programmes/en/lead/toolbox/Mixed1/TLU.htm

[9] Patrick, B.N., Rodrigue, A.B., Trésor, L.R., Dieudonné, W.S., Chance, A.B. and Espoir, B.B. (2019) Le système de production de la poule locale contraint son développement au Sud-Kivu, Est de la République Démocratique du Congo. Journal of Applied Biosciences, 135, 13821-13830. https://doi.org/10.4314/jab.v135i1.8

[10] Maass, B.L., Katunga, M.M.D., Chiuri, W.L., Gassner, A. and Peters, M. (2012) Challenges and Opportunities for Smallholder Livestock Production in Post-Conflict South Kivu, Eastern DR Congo. Tropical Animal Health and Production, 44, 1221-1232. https://doi.org/10.1007/s11250-011-0061-5

[11] Byarugaba, D.K. (2007) Poultry Sector Country Review. Emergency Centre for Transboundary Animal Diseases Socio Economics, Production and Biodiversity Unit. FAO Animal Production and Health Division and Makerere University, Uganda.

[12] Dhikusooka, M.T., Balikoowa, D., Mbattide, I., Beyihago, G., Ruhinda, J. and Kirunda, H. (2016) Chicken Manual on Increasing Productivity in Market Oriented Small Scale Chicken Producers. Poultry Research Institute, NIAS, RDA KAFACI Secretariat, ITCC, 300, Nongsaengymyeong-ro Wansan-gu Jeonju, 66 p.

[13] Ciewe C.S.A. (2016) Réussir en élevage de volailles, Ministère de l'élevage, Pêche et des Industries animales Poultry Research Institute, NIAS, RDA KAFACI Secretariat, ITCC, 300, Nongsaengymyeong-ro Wansan-gu Jeonju, 43p.

[14] Burkart, S., Contreras, A., Carolina Hölle, D., White, D., Peters, M. and Hoffmann, V. (2010) The Importance of Networking for Smallholder Swine and Chicken Producers in Colombia: A Social Network Analysis Tropentag. 2010 Conference on International Research on Food Security, Natural Resource Management and Rural Development, Zurich, 14-16 September 2010. https://doi.org/10.1016/S1353-4858(10)70136-7

[15] El Hadji, T. (2014) Revues nationales de l'élevage Secteur avicole Sénégal de la division de la production et de la santé animales de la FAO no7 Rome.

[16] Demeke, S. (2008) Poultry Sector Country Review of Ethiopia FAO. 